\title{
Recent DØ Results at Run II
}

\author{
Stefan Söldner-Rembold (on behalf of the D $\varnothing$ collaboration)* \\ Department of Physics and Astronomy, University of Manchester, Manchester, M13 \\ 9PL, United Kingdom
}

\begin{abstract}
The D $\varnothing$ experiment is taking data with an upgraded detector since March 2001. The integrated luminosity taken in Run II has now exceeded that taken in Run I. Selected physics results obtained with this data set are presented.
\end{abstract}

\section{Introduction}

The $\mathrm{D} \varnothing$ experiment is designed to study high energy proton-antiproton interactions at Fermilab's Tevatron collider in Batavia close to Chicago (USA). Protons $(\mathrm{p})$ and anti-protons $(\overline{\mathrm{p}})$ collide at a centre-of-mass energy $\sqrt{s}=1.96 \mathrm{TeV}$. A wide variety of physics topics can be studied at the Tevatron. Measurement of top-quark properties, electroweak precision variables and B-hadron properties including CP-violation and mixing, as well as searches for the Higgs boson, Supersymmetry (SUSY) and other phenomena beyond the Standard Model.

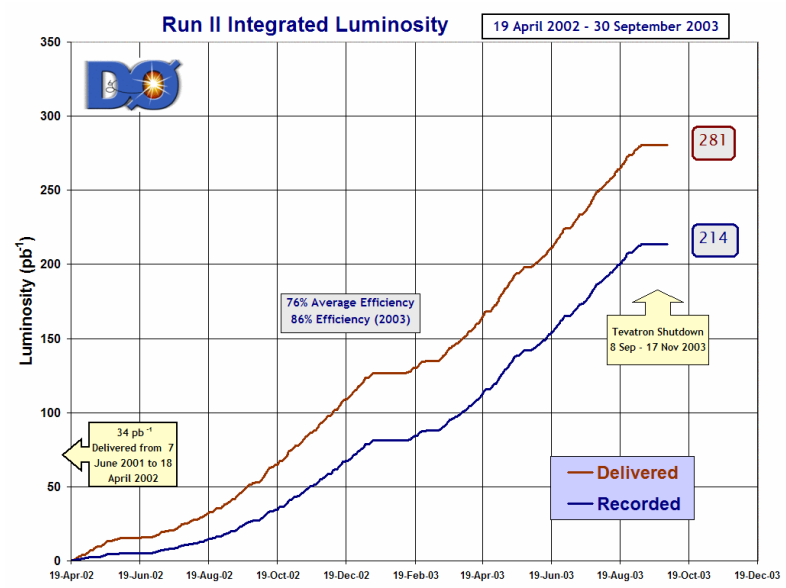

Fig. 1. Run II integrated luminosity for the period April 2002 - September 2003.

* submitted to the proceedings of the 4th International Conference on Physics Beyond the Standard Model, Beyond the Desert '03, Schloss Ringberg, Tegernsee, Germany, 9-14 June 2003 
The Run I data taking period lasted from 1992 to 1996 and produced many important results, among them the discovery of the top quark. Both the Tevatron and the detectors have been upgraded significantly in the following years. Compared to Run I the Tevatron's centre-of-mass energy $\sqrt{s}$ has increased from $1.8 \mathrm{TeV}$ to $1.96 \mathrm{TeV}$. The integrated luminosity at the end of Run II is expected to be a factor 50 higher than the Run I integrated luminosity of about $125 \mathrm{pb}^{-1}$.

The $\mathrm{D} \varnothing$ detector has also been upgraded significantly: Around the interaction region a new tracking system has been installed consisting of the Silicon Microvertex Tracker (SMT) and the Central Fibre Tracker (CFT). The CFT consists of eight axial layers of scintillating fibre aligned along the beam axis and eight layers of stereo fibres. The tracking detector is located within a 2 Tesla Solenoid. Further improvements include new preshower detectors surrounding the solenoid, the Forward Muon Detector, the Forward Proton Detector, and improved front-end electronics, trigger and data acquisition. The Liquid Argon Calorimeter, the Central Muon Detector and the muon toroid, which is used to provide a separate momentum measurement for the muons, are retained from Run I.
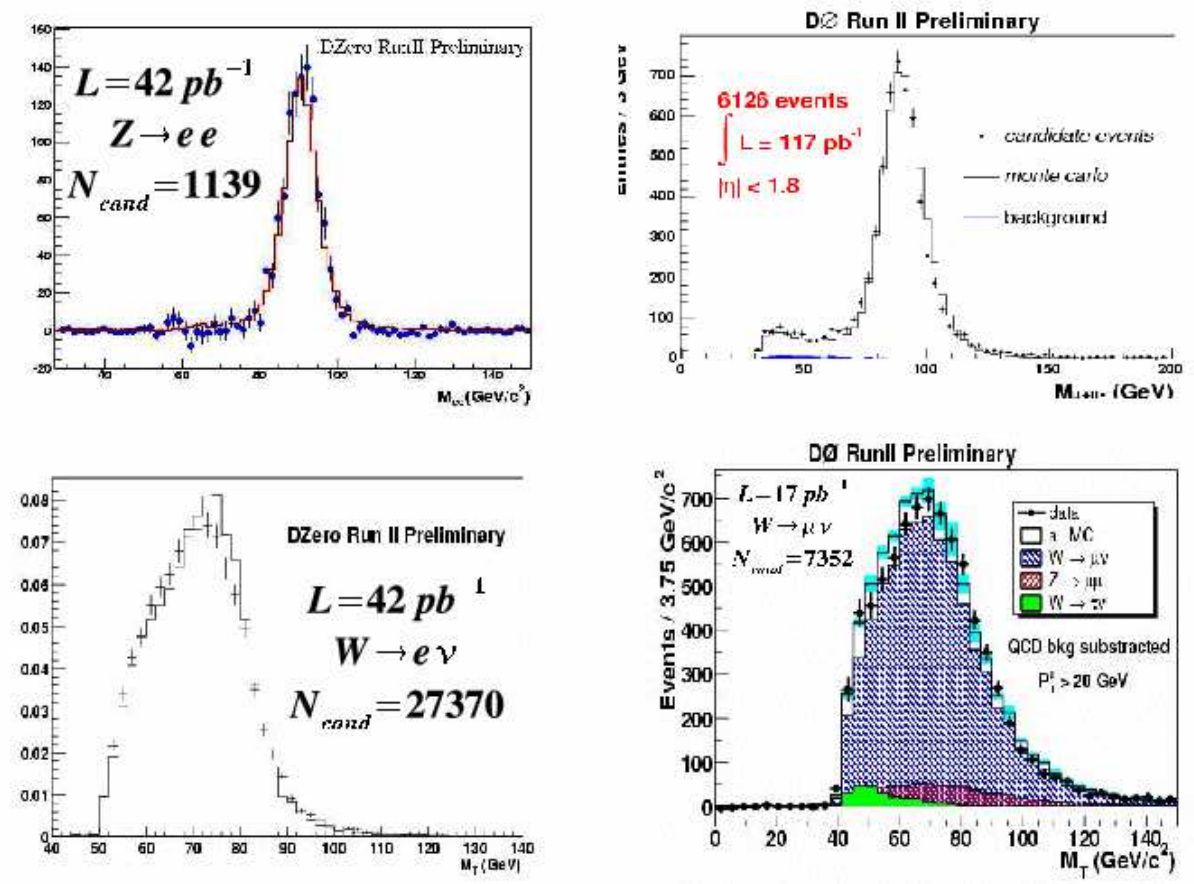

Fig. 2. top: invariant di-lepton mass for $\mathrm{Z} \rightarrow$ ee and $\mathrm{Z} \rightarrow \mu \mu$ candidate event; bottom: transverse mass for $\mathrm{W} \rightarrow \mathrm{e} \nu$ and $\mathrm{W} \rightarrow \mu \nu$ candidate events.

In this report a first look at the Run II data taken since April 2001 is presented. The Run II collected integrated luminosity is shown in Fig. 1. First a 
measurement of $\mathrm{W}$ and $\mathrm{Z}$ production is reported since it is both a fundamental physics measurement and a benchmark for the performance of the detector. Due to the topic of this conference the remaining presentation concentrates on searches for new physics beyond the Standard Model (Large Extra Dimensions, Leptoquarks and SUSY) and on the search for the Higgs Boson. In cases where the results have been updated since the conference, the updated results are being shown.

\section{Production of $\mathrm{W}$ and $\mathrm{Z}$ Bosons}

The measurement of the production of $\mathrm{W}$ and $\mathrm{Z}$ bosons is a central part of the $\mathrm{D} \emptyset$ physics programme. The long-term goal is to perform precision measurements of electroweak variables like the $\mathrm{W}$ mass and width, to study gauge couplings and to constrain the parton densities of the proton.

These processes are also important benchmarks for understanding the resolution and efficiency of the detector. The $\mathrm{W}$ and $\mathrm{Z}$ production cross-sections times branching ratios (BR) for the processes $\mathrm{p} \overline{\mathrm{p}} \rightarrow \mathrm{W}+\mathrm{X} \rightarrow \ell \nu+\mathrm{X}$ and $\mathrm{p} \overline{\mathrm{p}} \rightarrow \mathrm{Z}+\mathrm{X} \rightarrow \ell \ell+\mathrm{X}$ are measured for electron and muon final states $(\ell=\mathrm{e}, \mu)$. In the final Run II data sample we expect to observe more than $10^{5} \mathrm{Z}$ decays and more than $10^{6} \mathrm{~W}$ decays into electrons or muons. Since the theoretical cross-sections for $\mathrm{W}$ and $\mathrm{Z}$ production are calculable with high precision in the Standard Model, these events can also provide alternative measurements of the integrated luminosity with small experimental and theoretical errors.

The selection criteria for the $\mathrm{Z} \rightarrow \mu^{+} \mu^{-}$events require two oppositely charged muons with a transverse momentum greater than $15 \mathrm{GeV}$ within the pseudorapidity region $|\eta|<1.8$ where $\eta=-\ln \tan \theta / 2$. The di-muon invariant mass $m_{\mu \mu}$ must be greater than $30 \mathrm{GeV}$. Further cuts on the energy of tracks and calorimeter clusters in a halo around the muon direction are applied to ensure that the muons are isolated. The background contribution is estimated to be $(0.6 \pm 0.3) \%$. arising from $\mathrm{b} \overline{\mathrm{b}}$ production in QCD events and from cosmic rays and $(0.5 \pm 0.1) \%$ due to $\mathrm{Z} \rightarrow \tau^{+} \tau^{-}$events.

Table 1. Preliminary measurements of the cross-section times branching ratios for the different $\mathrm{W}$ and $\mathrm{Z}$ final states. The uncertainty due to the luminosity measurement is currently $10 \%$.

\begin{tabular}{|c|c|c|}
\hline \multicolumn{1}{|c|}{ Process } & $\sigma \cdot \mathrm{BR}$ & $\mathrm{L}$ \\
\hline $\mathrm{p} \overline{\mathrm{p}} \rightarrow \mathrm{Z}+\mathrm{X} \rightarrow \mu \mu+\mathrm{X}$ & $261.8 \pm 5.0($ stat $) \pm 8.9($ sys $) \pm 26.2($ lumi $) \mathrm{pb}$ & $117 \mathrm{pb}^{-1}$ \\
\hline $\mathrm{p} \overline{\mathrm{p}} \rightarrow \mathrm{Z}+\mathrm{X} \rightarrow \mathrm{ee}+\mathrm{X}$ & $294 \pm 11($ stat $) \pm 8($ sys $) \pm 29($ lumi $) \mathrm{pb}$ & $41.6 \mathrm{pb}^{-1}$ \\
\hline $\mathrm{p} \overline{\mathrm{p}} \rightarrow \mathrm{W}+\mathrm{X} \rightarrow \mu \nu+\mathrm{X}$ & $3226 \pm 128($ stat $) \pm 100($ sys $) \pm 323($ lumi $) \mathrm{pb}$ & $17.3 \mathrm{pb}^{-1}$ \\
\hline $\mathrm{p} \overline{\mathrm{p}} \rightarrow \mathrm{W}+\mathrm{X} \rightarrow \mathrm{e} \nu+\mathrm{X}$ & $3054 \pm 100($ stat $) \pm 86($ sys $) \pm 305($ lumi $) \mathrm{pb}$ & $41.6 \mathrm{pb}^{-1}$ \\
\hline \hline
\end{tabular}


Events with $\mathrm{W} \rightarrow \mu \nu$ decays are selected by requiring exactly one muon with a transverse momentum greater than $20 \mathrm{GeV}$. The muon must be isolated and additional cuts against muons from cosmic rays are applied. The missing transverse energy in the event, calculated from the calorimeter information and corrected for the muon transverse momentum, must exceed $20 \mathrm{GeV}$. About $17 \%$ of the selected events are expected to be background, mainly from $\mathrm{Z} \rightarrow \mu \mu$, $\mathrm{W} \rightarrow \tau \nu$ and $\mathrm{QCD}$ events.

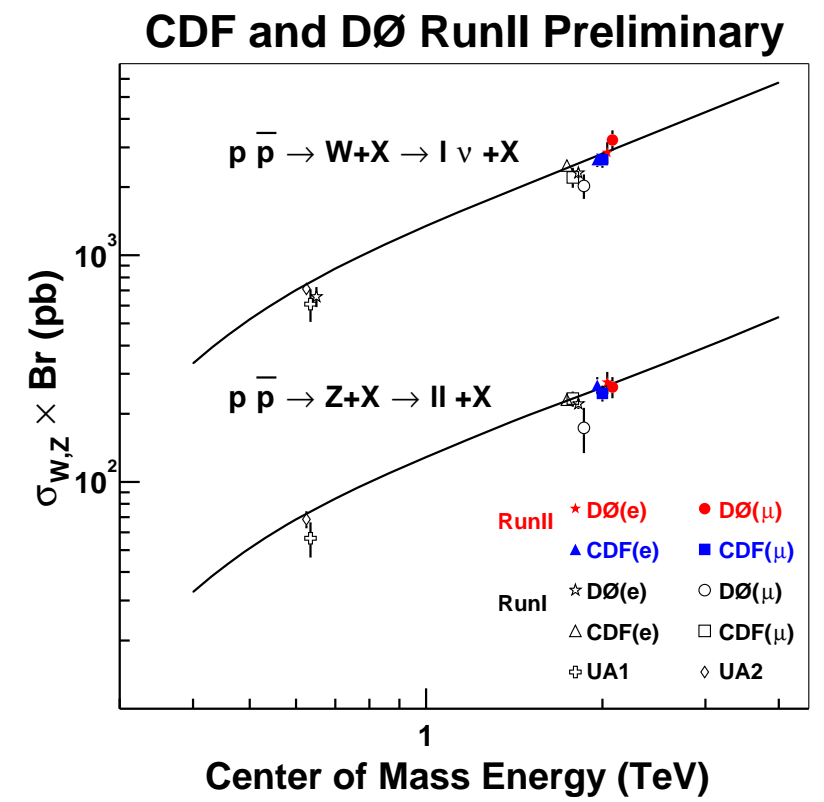

Fig. 3. Summary plot for the $\mathrm{W}$ and $\mathrm{Z}$ production cross-section in electron and muon final states as a function of $\sqrt{s}$.

The identification of electrons in $\mathrm{W} \rightarrow \mathrm{e} \nu$ and $\mathrm{Z} \rightarrow$ ee events is based on requiring one or two electrons with a transverse momentum of more than 25 $\mathrm{GeV}$ in the central calorimeter $(|\eta|<1.1)$. For the $\mathrm{W} \rightarrow \mathrm{e} \nu$ channel the missing transverse energy due to the neutrino must be larger than $25 \mathrm{GeV}$.

Figure 2 shows the di-lepton invariant mass for the $\mathrm{Z}$ candidates and the transverse mass for the $\mathrm{W}$ candidates. The cross-sections are given Table 1 . In Figure 3, the measured cross-sections are compared with the cross-sections measured by the CDF collaboration and with the cross-sections measured at lower $\sqrt{s}$. Within the uncertainties good agreement is observed with the evolution of the cross-section expected from a NLO calculation [1]. 


\section{Searches for New Physics}

The $\mathrm{D} \varnothing$ experiment has started to search for rare event topologies which are not consistent with the expectations of the Standard Model. Most searches are based on leptons (mainly electrons and muons) and on missing transverse energy due to neutrinos. Similar topologies can be interpreted in many different models which predict new physics beyond the Standard Model. With the current integrated luminosity some of the searches start to become competitive with similar searches performed at Run I, at LEP and at HERA. In this report only a few highlights can be presented.

\subsection{Large Extra Dimensions}

It has been suggested in the context of string theory that the apparent Planck scale, $M_{\mathrm{Pl}} \simeq 10^{19} \mathrm{GeV}$, which is the scale where gravity becomes comparable in strength to other interactions, can be related to physics at the $\mathrm{TeV}$ scale and is therefore accessible to experimental tests at current high energy experiments. Arkani, Dimopoulos, and Dvali 2] have proposed a framework where the hierarchy problem is solved by assuming that the fundamental Planck scale $M_{\mathrm{S}}$ is similar in size to the electroweak scale. This is accomplished by relating the

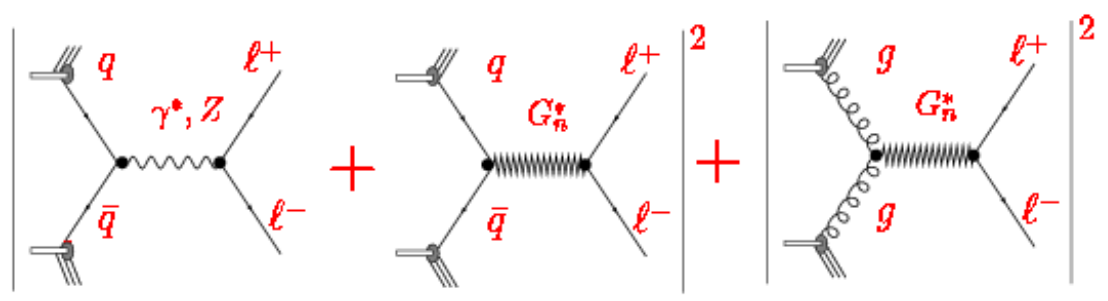

Fig. 4. Diagrams for di-lepton production in the presence of Large Extra Dimensions [3].

apparent Planck scale $M_{\mathrm{Pl}}$ to the Planck scale in a $(3+n)$-dimensional theory,

$$
M_{\mathrm{Pl}}=\left(M_{\mathrm{PL}}^{(3+n)}\right)^{n+2} R^{n}=M_{\mathrm{S}}^{n+2} R^{n},
$$

where $n$ is the number of Extra Dimensions and $R$ is the compactification radius. Assuming for the fundamental Planck scale $M_{\mathrm{S}} \simeq 1 \mathrm{TeV}, n=2$ corresponds to $R \simeq 1 \mathrm{~mm}$, which is accessible to gravity experiments. Only accelerator experiments, however, can study the region $n>2$.

Large Extra Dimensions are expected to manifest themselves through the effects of virtual graviton exchange in di-fermion or di-boson production (see Fig. (4). They are parametrised via a single variable $\eta_{G}=\mathcal{F} / M_{\mathrm{S}}^{4}$, where $\mathcal{F}$ is a dimensionless parameter of order unity. Different formalisms are used to define $\mathcal{F}$. 

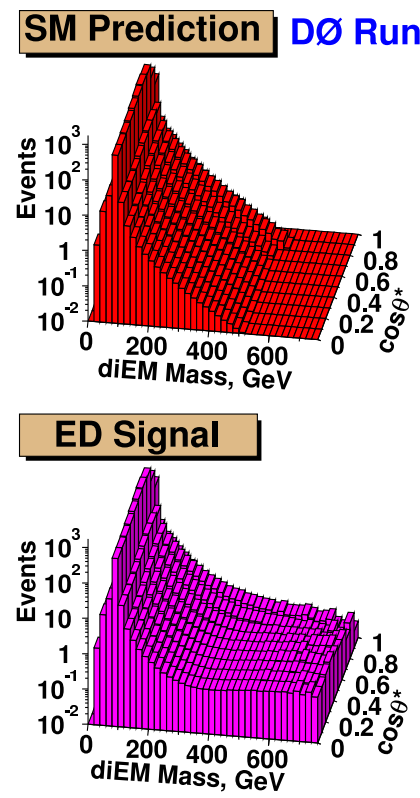

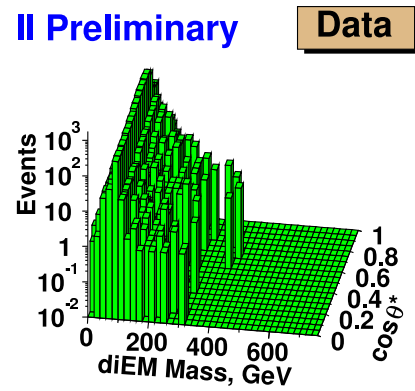

QCD Background

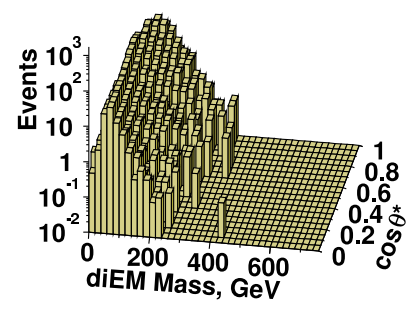

Fig. 5. Two-dimensional $\left(M, \cos \theta^{*}\right)$ distributions for the ee and $\gamma \gamma$ final states. The expected Standard Model (SM) background, the expected signal due to Large Extra Dimensions (ED) for $\eta_{G}=2$, the QCD background and the data are shown separately.

The cross-section is parametrised by

$$
\frac{\mathrm{d}^{2} \sigma}{\mathrm{d} M \mathrm{~d} \cos \theta *}=f_{\mathrm{SM}}+f_{\text {interference }} \eta_{G}+f_{\text {Kaluza-Klein }} \eta_{G}^{2}
$$

where $f$ are functions of the di-fermion (or di-boson) invariant mass $M$ and the scattering angle $\cos \theta^{*}$ in the centre-of-mass frame. The first term is due to the Standard Model, the last term due to Kaluza-Klein graviton exchange and the second term due to interference. Graviton exchange would modify the $\left(M, \cos \theta^{*}\right)$ distributions.

A search for Large Extra Dimensions in the di-electron and di-photon channels has been performed using $L=130 \mathrm{pb}^{-1}$ of data. A similar analysis has been performed in the di-muon channel using $100 \mathrm{pb}^{-1}$ of data. For the di-electron and di-photon analysis two electromagnetic objects in the calorimeter, which are consistent with an electron or photon hypothesis and have transverse energies greater than $25 \mathrm{GeV}$, are required for the final selection. The two-dimensional distribution of the invariant mass $\mathrm{M}$ and the scattering angle $\cos \theta^{*}$ is shown in Fig. 5

New limits on the fundamental Planck scale $M_{\mathrm{S}}$ of $1.28 \mathrm{TeV}$ (in the GRW convention [4]) are derived from a fit to the $\left(M, \cos \theta^{*}\right)$ distribution, exceeding limits obtained with similar statistics in Run I [5]. Combined with the published 
Run I result, this corresponds to a lower limit of $M_{\mathrm{S}}>1.37 \mathrm{TeV}$, which is the most stringent limit on Large Extra Dimensions to date.

\section{$3.2 \quad$ Leptoquarks}

Leptoquarks (LQ) are coloured spin 0 or spin 1 particles carrying both baryon (B) and lepton (L) quantum numbers. They appear in many extensions of the Standard Model as a consequence of the symmetry between the lepton and quark sectors. The Buchmüller-Rückl-Wyler (BRW) model [6] assumes lepton and baryon number conservation. Moreover the simplifying assumption is made that a given leptoquark couples to just one family of fermions. Leptoquarks may decay into either a charged lepton and a quark or into a neutrino and a quark. The branching ratio of the decay into a charged lepton and a quark is commonly denoted by $\beta$.
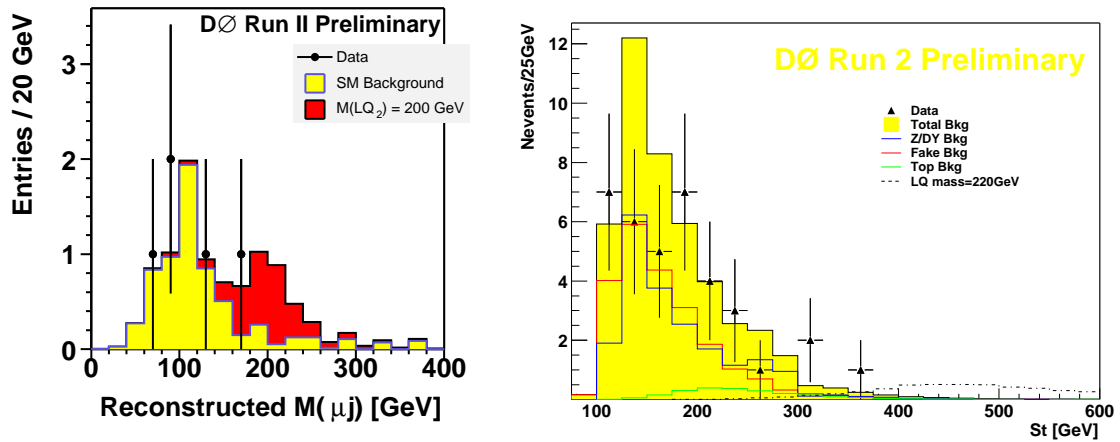

Fig. 6. left plot: Reconstructed $\mu+$ jet mass for all di-muon+di-jet events with $M(\mu \mu)>110 \mathrm{GeV}$. There are two possibilities to combine the two highest- $p_{\mathrm{T}}$ muons with the two highest transverse energy jets. Only the combination with the smaller mass difference of the two leptoquark candidates of the event is chosen, and the reconstructed LQ mass of this event is the mean of the masses of the two $\mu+$ jet systems; right plot: $S_{T}$ distribution of di-electron + di-jet events in data (triangles) compared to background (histogram) after applying a veto cut against $\mathrm{Z}$ events. The dashed histogram is the $S_{T}$ distribution for a $220 \mathrm{GeV}$ LQ signal.

The $\mathrm{D} \emptyset$ experiment has searched for scalar (spin 0) first generation and second generation leptoquarks decaying into eq and $\mu \mathrm{q}$ final states, respectively. The scalar leptoquarks are pair produced. The mass limits are therefore independent of the Yukawa coupling $\lambda$. Assuming $\beta=1$ the final states are eeqq for the first generation and $\mu \mu \mathrm{qq}$ for the second generation leptoquark. Both analyses therefore require at least two jets with a transverse energy of more than $25 \mathrm{GeV}$ in addition to two charged leptons with high transverse momentum $p_{\mathrm{T}}$.

The reconstructed $\mu+$ jet mass distribution is shown in Fig. [6 together with a simulated second generation LQ signal with a mass of $200 \mathrm{GeV}$. The scalar sum 
$S_{T}$ of the transverse energy of the two electrons and the two leading jets in the eeqq channel is found to give a good separation of signal and background. The $S_{T}$ distribution is shown in Fig. [ together with a simulated first generation LQ signal with a mass of $210 \mathrm{GeV}$. No evidence of LQ production is observed in any of the channels,

A data sample of $135 \mathrm{pb}^{-1}$ is used for the eeqq channel. A lower mass limit of $231 \mathrm{GeV}$ for a first generation scalar leptoquark at 95\% Confidence Level (CL) is obtained, in the case of $\beta=1$. The combined mass limit for a scalar first generation leptoquark is $253 \mathrm{GeV}$ from D $\varnothing$ Run II and Run I results, which is the most stringent limit to date.

The integrated luminosity used for the $\mu \mu \mathrm{qq}$ channel is $104 \mathrm{pb}^{-1}$. No excess of the data over the expected background is found, and the 95\% CL mass limit for a scalar second generation leptoquarks assuming $\beta=1$ is found to be $186 \mathrm{GeV}$.

In addition, the case where one of the two leptoquarks decays into an electron and a quark and the other in a neutrino and a quark is also considered. Assuming $\beta=0.5$, an upper mass limit of $156 \mathrm{GeV}$ at $95 \%$ CL is obtained for a data set with an integrated luminosity of $121 \mathrm{pb}^{-1}$.

\subsection{Gauge Mediated Supersymmetry (GMSB)}

$\mathrm{D} \varnothing$ is searching for supersymmetric particles in a variety of different theoretical frameworks. A particularly interesting and clear signature is provided by Gauge Mediated Supersymmetry Breaking (GMSB). In GMSB models, the gravitino $(\tilde{G})$ is the lightest supersymmetric particle (LSP).

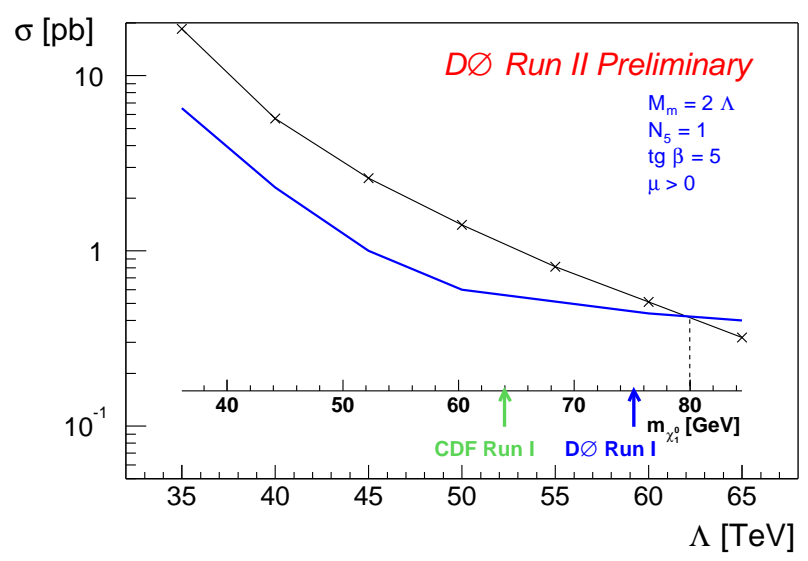

Fig. 7. GMSB cross-section as a function of the SUSY breaking scale $\Lambda$ for $\tan \beta=5$, where $\tan \beta$ is the ratio of the Higgs vacuum expectation values. The $95 \%$ confidence levels limits and the Run I CDF and D $\varnothing$ results are also shown [7]. 
The neutralino is the next-to-lightest supersymmetric particle (NLSP) in the scenario considered here. A typical process within this scenario is

$$
\mathrm{p} \overline{\mathrm{p}} \rightarrow \tilde{\chi}_{1}^{0} \tilde{\chi}_{1}^{0}+\mathrm{X} \rightarrow \gamma \gamma+\tilde{G} \tilde{G}+X
$$

where the gravitino $\tilde{G}$ remains undetected.

An inclusive search for signals of new physics is therefore carried out in the diphoton plus missing energy channel. The analysis requires two electromagnetic clusters in the central part of the calorimeter with a transverse energy greater than $20 \mathrm{GeV}$ and a missing transverse energy greater than $35 \mathrm{GeV}$. In total $1.38 \pm 0.30$ events are expected from the Standard Model background and zero events are observed. The GMSB cross-section as a function of the SUSY breaking scale $\Lambda$ is shown in Fig. 7 In the absence of a signal, lower bounds of $m_{\tilde{\chi}_{1}^{0}}>$ $80 \mathrm{GeV}$ and $m_{\tilde{\chi}_{1}^{ \pm}}>144 \mathrm{GeV}$ are derived for the neutralino and chargino mass, respectively.

\section{Searches for the Higgs Boson}

The search for the Higgs boson is one of the most ambitious physics goals of the Tevatron. Production of the Standard Model (SM) Higgs boson through the Higgs-strahlung processes, $\mathrm{qq}^{\prime} \rightarrow \mathrm{W}^{*} \rightarrow \mathrm{WH}$ and $\mathrm{q} \overline{\mathrm{q}} \rightarrow \mathrm{Z}^{*} \rightarrow \mathrm{ZH}$, where the $\mathrm{W}$ and $\mathrm{Z}$ boson decay leptonically, is the most promising search channel at the Tevatron (Fig. 8). The SM cross-section times branching ratio for producing a Higgs with a mass of $120 \mathrm{GeV}$ is about $30 \mathrm{fb}$ for the $\mathrm{WH}$ process if the W bosons decays into electron or muons. The signal rate in the current data sample corresponding to an integrated luminosity of about $200 \mathrm{pb}^{-1}$ is therefore much too small to be observed.

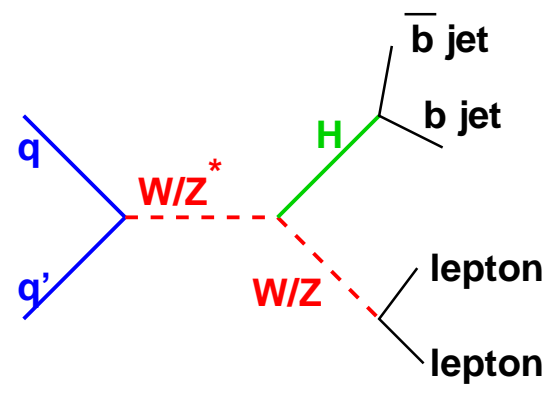

Fig. 8. Higgs production in the channel $\mathrm{qq}^{\prime} \rightarrow \mathrm{W}^{*} \rightarrow \mathrm{WH}$.

The "Road to the Higgs Discovery", however, is well defined. Searching for the SM Higgs boson in the WH (or $\mathrm{ZH}$ ) channel first requires a precise understanding of the production of $\mathrm{W}$ and $\mathrm{Z}$ bosons. These results have been presented in Section 2. 

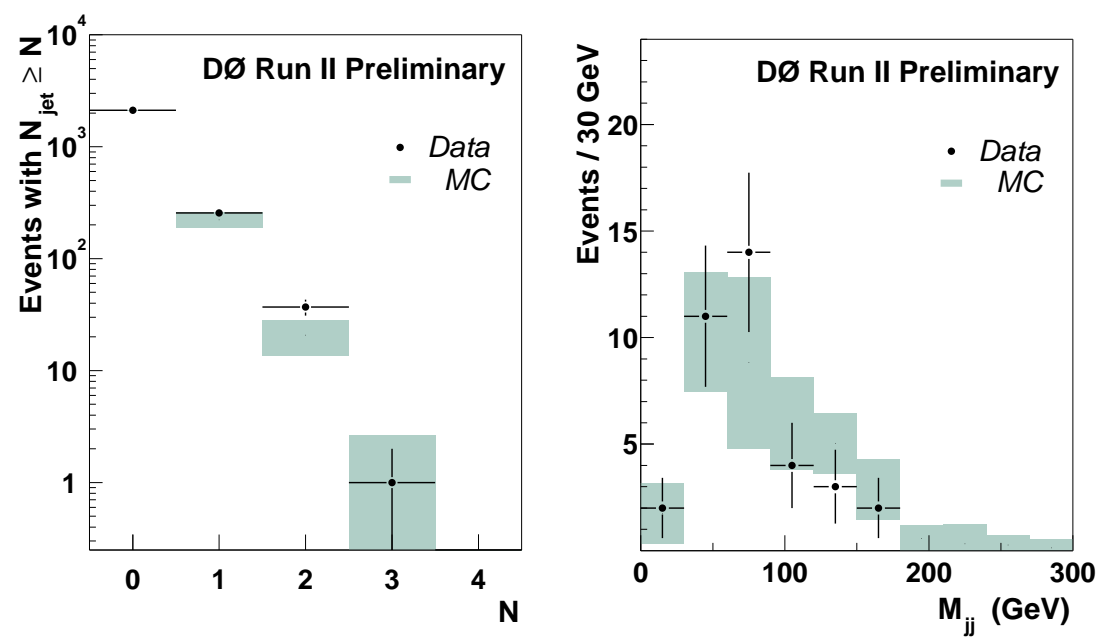

Fig. 9. The data points show the combined data for $\mathrm{Z}$ events with e+jets and $\mu+$ jets final states. The shaded bands show the Monte Carlo prediction. The width of the band reflects the (currently statistics limited) range allowed by the jet energy calibration. left plot: inclusive jet multiplicities; right plot: di-jet invariant mass.

The next step is related to understanding (di-)jet production in events with $\mathrm{W}$ and $\mathrm{Z}$ production. In Fig. 9 the measured jet multiplicity and di-jet invariant mass in $\mathrm{Z} \rightarrow \mu \mu$ and $\mathrm{Z} \rightarrow$ ee events are compared to a simulation using the PYTHIA Monte Carlo program. The jet selection requires the jet to have a transverse momentum larger than $20 \mathrm{GeV}$ and it has to lie within the pseudorapidity range $|\eta|<2.5$. The band used for the Monte Carlo prediction shows the uncertainty due to the calorimeter energy scale. Reasonably good agreement is observed between data and Monte Carlo.

For SM Higgs mass below $135 \mathrm{GeV}$, the Higgs boson primarily decays into b $\bar{b}$ final states. Jets originating from b-quarks can be identified using the lifetime $\tau \simeq 1.5 \mathrm{ps}$ of $\mathrm{B}$ hadrons which translates into a decay length of the order $1 \mathrm{~mm}$ in the detector. The tagging of b-jets is performed by measuring the impact parameter of tracks, the reconstruction of secondary vertices and by identifying semi-leptonic b decays. An important tool is the Silicon Microvertex Detector (SMT) which has been shown to measure the impact parameter of tracks with large transverse momentum with a resolution of $\simeq 15 \mu \mathrm{m}$.

If the mass of the Higgs particle is in the range of 135 to $200 \mathrm{GeV}$, it will predominantly decay to $\mathrm{W}$ pairs. A search for Higgs decays in $\mathrm{W}$ pairs has been performed for decays into ee, $\mathrm{e} \mu$ and $\mu \mu$ final states using data corresponding to an integrated luminosity of $118 \mathrm{pb}^{-1}$. The electrons have to be within a pseudorapidity range of $|\eta|<1.3$ and the muons within $|\eta|<2$. The cuts on the transverse momenta vary between $7 \mathrm{GeV}$ and $20 \mathrm{GeV}$ depending on the final state and the Higgs mass studied. For the $\mu \mu$ channel the azimuthal angle 

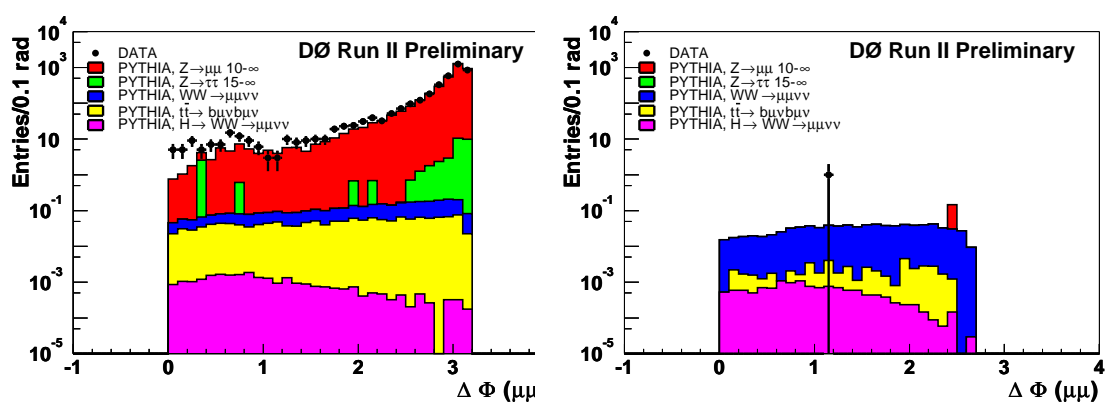

Fig. 10. Azimuthal angle between the two muons, $\Delta \phi(\mu \mu)$ after a loose pre-selection and after the final cuts.

between the two muons, $\Delta \phi(\mu \mu)$, is shown in Fig. 10. Since the spins of the two W bosons from the Higgs decay are correlated, $\Delta \phi(\mu \mu)$ is on average smaller for signal than for background events.

After the final event selection using a sequence of cuts to suppress background one event is observed in good agreement with the Monte Carlo expectation for the sum of all SM processes of $0.95 \pm 0.12$ events. Since no signal is observed in this channel and in the e $\mu$ and ee channels, a combined limit on the Higgs production cross section times the branching ratio $\mathrm{BR}(H \rightarrow W W)$ at $95 \%$ CL is obtained in Fig. 11] A SM Higgs boson in the WW decay channel cannot be excluded yet with the current integrated luminosity, but the sensitivity is within less than a factor of ten for a 4 th generation model.

In addition to the results presented here the $\mathrm{D} \varnothing$ experiment is searching for Higgs production in several other scenarios beyond the Standard Model. Results have recently been presented on Higgs decays into two photons and on doublycharged Higgs bosons decaying into two muons.

\section{Summary}

First results of the $\mathrm{D} \varnothing$ experiment on $\mathrm{W}$ and $\mathrm{Z}$ production, searches for Large Extra Dimensions, Leptoquarks and Gauge Mediated Supersymmetry have been reported. With the recorded integrated luminosity many search results are already improving on previous limits obtained at Run I and LEP. The Tevatron is currently the only running collider at the high energy frontier and many exciting results are expected in future years.

\section{Acknowledgement}

I would like to thank Hans Volker Klapdor-Kleingrothaus and Irina Krivosheina for the excellent organisation of the scientific and social aspects of the conference. The wide variety of topics presented at the conference and the beautiful setting of Schloss Ringberg made the conference a most enjoyable experience. I also 


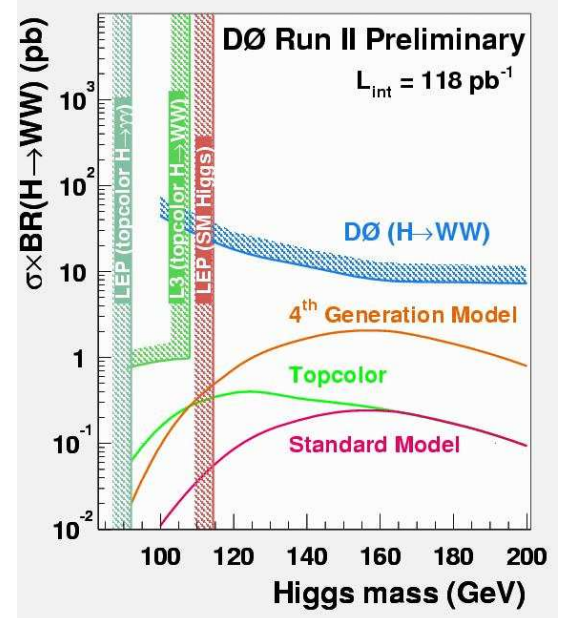

Fig. 11. Higgs production cross section times $\mathrm{BR}(H \rightarrow W W)$ excluded with the current DØ data (and LEP experiments), along with the expectations from various models. This result is a combination of the ee, $\mathrm{e} \mu$ and $\mu \mu$ analyses.

want to thank my colleagues at $\mathrm{D} \varnothing$ for their help in preparing this report and Emily Nurse for her careful reading of the manuscript.

\section{References}

1. C.R. Hamberg, W.L. van Neerven and T. Matsura, Nucl. Phys. B359, 343 (1991)

2. N.Arkani-Hamed, S. Dimopoulos and G. Dvali, Phys. Lett. B429, 263 (1998); Phys. Rev. D59, 086004 (1999); L. Antoniadis, N.Arkani-Hamed, S. Dimopoulos and G. Dvali, Phys. Lett. B436, 257 (1998).

3. A. Gupta, N. Mondal and S. Raychaudhuri, hep-ph/9904234

4. G. Giudice, R. Rattazi and J. Wells, Nucl. Phys. B544, 3 (1999) and hep-ph/9811291

5. B. Abbott et al., Phys. Rev. Lett. 86, 1156 (2001).

6. W. Buchmüller, R. Rückl and D. Wyler, Phys. Lett.B191, 442 (1987).

7. F. Abe et al., Phys. Rev. D59, 092002 (1999); B. Abbott et al., Phys. Rev. Lett. 80, 442 (1998). 